\title{
ELEMENTOS LINGÜÍSTICOS QUE OBSTACULIZAN EL APRENDIZAJE DE LOS NOMBRES DE LOS NÚMEROS NATURALES EN MÍSKITU
}

Víctor Winsing Castrillo ${ }^{[8]}$ y Alejandro Torres ${ }^{[9]}$

\section{Resumen}

Este estudio se efectuó en el segundo semestre de 2009, el objetivo fue identificar los elementos lingüísticos y la base de numeración que favorecen el proceso enseñanza de los nombres de números naturales del 1 al 100 en la lengua miskitu, en la escuela primaria bilingüe "Simeón Walls", comunidad de Kuamwatla, municipio Prinzapolka, RAAN.

Es un estudio cualitativo, descriptivo y de corte transversal. El universo de estudio lo constituyó la comunidad de 12 docentes y 255 estudiantes de la escuela en mención. La muestra utilizada fueron 10 por ciento de estudiantes, 6 por ciento de docentes y 10 miembros de la comunidad. Para el procesamiento y análisis de la información se elaboró una matriz de cada instrumento utilizado, y a partir de ello se procedió a la redacción del informe de los resultados.

Entre los resultados se destacan los siguientes: i) existen cambios en los nombres de los numeros naturales, ii) evidentes dificultades lingüísticas en los nombres de los números naturales del miskitu y iii) los nombres de los números naturales del sistema de numeracion miskitu en uso actual, tiene indicio de subbase quinaria y base vigesimal.

En base a los resultados del estudio, se realizó una propuesta de mejoras de lexemas, morfemas, alomorfos, ortográficos, sintaxis y de fonemas en los nombres de los números naturles de miskitu o a 100, desde el punto de vista etimológico combinado con neologismos.

Palabras claves: Lengua miskitu, números, elementos obstaculizadores.

\section{Introducción}

Enseñar en mískitu los contenidos de matemática es difícil, especialmente el vocabulario técnico matemático en el cual no hay expresiones equivalentes que se conozcan

[8] Lic. Educación Intercultural Bilingüe, URACCAN BILWI, Kumwatla-Prinzapolka, victorwinsin@yahoo.es

[9] Lic. Matematica, URACCAN Bilwi, Bo. Filimon Rivera, walpa5@yahoo.com 
en la lengua materna. Enseñar los números naturales de 1 al 100 es toda una odisea a paratir de que la mayoría de los docentes no conocen los nombres en mískitu de los números naturales en forma sistemática, a esto hay que agregarle que la lengua miskitu tiene muchos vocablos con diferentes interpretaciones de acuerdo a cada comunidad: miskitu mam, mískitu wanky, mískitu tawira.

Por otra parte no hay una base de sistema numérico bien definido que permita enseñar los nombres de los números naturales del uno al cien y que sea extensible lógicamente en el proceso enseñanza de los números mayores que cien. Esto nos permite preguntarnos ¿será que no hemos encontrado los elementos lingüísticos adecuados que nos permita enseñar de manera lógica los nombres de los numerales naturales del 1 al cien?

Asimismo, se puede decir que los docentes conocen algunos nombres de los números, pero no todos, debido a que en miskitu son muy largos, repetitivos y con sonidos complejos. Esto induce a que los docentes hagan una mezcolanza: miskitu, creol yespañol en la forma de explicar los nombres de los nùmeros. Las anteriores dificultades son el motivo de esta investigación.

\section{Revision de Literatura}

Según URACCAN (2001:31), se plantea que: "los cambios lingüísticos, se debe a causas de intralingüística o extralingüística; intralingüística es el cambio de la etimología de una palabra, por los mismos hablantes". Y extralingüístico, este se produce con mayor rapidez cuando hay un factor historico-cultural crítico (invasión, conquista, revolucion, etc), cuando el ambito historico-cultural es intrascendente, el cambio se produce muy lentamente.

Tambien "los cambios lingüísticos se debe a causa de las palabras vulgares, que son todo término, giros, dichos o frases mal empleadas, mal pronunciadas o escritas" (MECD, 2004:139).

\section{Elementos lingüísticos}

Según URACCAN (2001; 29) se define que los elementos lingüísticos "son los elementos de la lengua que hace posible el uso de la lengua en un contexto, especialmente en lo fónico, morfológico, ortográfico, sintactico y etimológico".

A continuación los conceptos de los diferentes elementos lingüísticos, que abarca en este estudio: 
Fonemas: "son los sonidos del lenguaje hablado. También aplica a cómo se pronuncia todo la escritura cuyas letras representan sonidos y como se diferencia". (htt.www. lablaa.org/ayudatectareas/español/espa-16.htm.)

Morfemas: "son cada una de las partes más pequeñas con significados, en que se puede dividir de una palabra”. Según http//htm-rincón del vago.com/morfologico-1htm

Alomorfos: "son cada una de las variantes de un morfema que tiene significado idéntico. Ejemplo -s, -es son alomorfos del morfema de plural en español”. (Real Academia Española, 2006:74).

Lexema: (raíz) según Matus (2007:101) "es la parte de una palabra, que no sufre alteración en todos los procesos de modificación. Y quien nos puede decir de su origen".

Ortografía: "es la que enseña a escribir correctamente las palabras. Y es un conjunto de reglas que indican como escribir, acentuar las palabras y como se utilizan algunas letras". (MED, 1987: V).

Etimología: "es el origen (historia) o derivación de las palabras". (MED, 1987: VI)

Neologismo: "son las palabras, expresiones, acepciones de creación recientes, que aparece o se adopta en una lengua”. (Real Academia Española, 2006:1023).

Sintaxis: "es la que se encarga de estudiar las correctas coordinaciones, ordenamientos o enlazamientos de las palabras en los enunciados". (Real Academia Española, 2006:1369).

\section{Algunas Bases de sistemas de numeraciones estudiadas}

Quinario: "Sistema de numeración de base quinario, es el sistema de numeración que tiene por base el numero 5, es el sistema compuesto de cinco elementos, unidades, signos o símbolos que expresan cantidades. Posee cinco símbolos: o, 1, 2, 3,4. Este sistema tiene su origen en el hecho de que los humanos tienen cinco dedos en cada mano. Aún así, cinco es un número primo, y por ello el sistema quinario se utiliza como sub-base del sistema decimal y el vigesimal".

Vigesimal: "Los mayas idearon un sistema de base 20 con el 5 cómo base auxiliar. La unidad se representaba por un punto. Dos, tres, y cuatro puntos servían para 2, 3 y 4 . El 5 era una raya horizontal, a la que se añadían los puntos necesarios para represen$\operatorname{tar} 6,7,8$ y 9 . Para el 10 se usaban dos rayas, y de la misma forma se continúa hasta el 20, con cuatro rayas". 


\section{Numeración miskita}

En el cuadro No. 1 se presenta la numeración en lengua Miskitu según la Sociedad Bíblica (1974) y Cárdenas y David (1993).

Cuadro No. 1: Numeración en lengua Miskitu según dos fuentes consultadas.

\begin{tabular}{clc}
\hline No. & Cárdenas y David (1993) & Sociedad Bíblica (1974) \\
\hline 0 & Apu & \\
\hline 1 & Kumi & Kum \\
\hline 2 & Wal & Wol \\
\hline 3 & Yumhpa & Yumpa \\
\hline 4 & Walhwal & Wolwol \\
\hline 5 & Matsip & - \\
\hline 6 & Matlalkahbi & - \\
\hline 7 & Matlalkahbi pura kumi & - \\
\hline 8 & Matlalkahbi pura wal & - \\
\hline 9 & Matlalkahbi pura yumhpa & - \\
\hline 20 & - & Yawanaiska \\
\hline
\end{tabular}

Sin embargo, Urbina (2004:51,52), al respecto refiere que: "en el idioma mîskitu existen dos posibilidades de números cardinales; una autóctona que casi esta en desuso y la otra de origen ingles que sustituyó a la primera en uso", este es el siguiente:

\begin{tabular}{lc}
\hline \multicolumn{1}{c}{ Autóctona } & Origen ingles \\
\hline Kum (i) & Wan \\
\hline Wal & Tu \\
\hline Yumhpa & Tri \\
\hline Walhwal & Pur \\
\hline Matsip & Paip \\
\hline Matlalkahbi & Siks \\
\hline Matlalkahbi pura kum & Sibin \\
\hline Matlalkahbi pura wal & It \\
\hline Matlalkahbi pura yumhpa & Nain \\
\hline Matawalsip & Tin \\
\hline Dusa kum & Andat \\
\hline
\end{tabular}




\begin{tabular}{lc}
\hline \multicolumn{1}{c}{ Autóctona } & Origen ingles \\
\hline Dusa wal & Tu andat \\
\hline Lal bakriki kum & Paip andat \\
\hline Lal kum & Tausin \\
\hline
\end{tabular}

\section{Materiales y Métodos}

En los aspectos metodológicos, es un estudio cualitativo, descriptivo de corte transversal. El universo de estudio lo constituyó la comunidad de 12 docentes y 255 estudiantes de la escuela en mención. La muestra utilizada fue el 10\% de estudiantes, $6 \%$ de docentes y 10 miembros relevantes de la comunidad entre ancianos, padres y madres de familias. Como criterio de selección se contempló ser estudiante o docentes activo, ser anciano líder de la comunidad y ser padre o madre de familia con estudiantes activos.

Para el proceso de levantado de la información se aplicaron pruebas diagnósticas a los estudiantes, entrevistas a docentes y entrevistas a grupos focales con padres y madres de familias, así con ancianos líderes. En lo referente al aspecto ético se garantizó el anonimato de los informantes y la devolución de los resultados a la comunidad educativa. Para el procesamiento y análisis de la información se elaboró una matriz de cada instrumento, se realizó una descripción de las respuestas concretas y realidades encontradas, y a partir de ello se procedió a la redacción del informe de la investigación de los resultados.

\section{Resultados y Discusiones}

Después de haber realizado el trabajo, consideramos haber logrado una gran experiencia y aprendizaje, que indudablemente deja constancia de las multiples necesidades por analizar y profundizar en el campo educativo, específicamente en las escuelas donde se desarrollan los programas de Educación Intercultural Biingüe.

Acontinuación los resultados más relevantes:

\section{Los cambios en los nombres de los números naturales de mîskitu, a través de los tiempos}

Si comparamos los cuadros anteriores, los nombres de los números naturales de miskitu en 1974, estaba más cerca al original, pero de 1993 hasta hoy, hay más cambios. 


\section{REVITALIZACIÓN LINGÜÍSTICA Y CULTURAL}

Cuadro No. 2. Dificultades lingüísticas más significativas en los nombres de los números naturales tipicos del miskitu.

\begin{tabular}{|c|c|c|c|}
\hline No & Docentes & Estudiantes & $\begin{array}{c}\text { Dificultades más } \\
\text { relevantes }\end{array}$ \\
\hline O & Apu 100 & $\begin{array}{c}\text { Apu } \\
\text { Ahpu }\end{array}$ & Ortográfico \\
\hline 3 & $\begin{array}{l}\text { Yumhpa } \\
\text { Yuhmpa }\end{array}$ & $\begin{array}{l}\text { Yumhpa } \\
\text { Yuhmpa }\end{array}$ & Ortográfico \\
\hline 4 & $\begin{array}{l}\text { Walhwal } \\
\text { Walhwalh }\end{array}$ & $\begin{array}{l}\text { Walhwal } \\
\text { Walhwalh }\end{array}$ & Ortográfico \\
\hline 5 & $\begin{array}{l}\text { Matsip } \\
\text { Matchip }\end{array}$ & & Morfema \\
\hline 6 & Matlalkahbi & $\begin{array}{c}\text { Matlalkahbi } \\
\text { Matsip pura kumi }\end{array}$ & Etimológico \\
\hline 7 & $\begin{array}{l}\text { Matlalkahbi pura kumi } \\
\text { Matlal kahbi pura kumi }\end{array}$ & & Alomorfos \\
\hline 8 & Matlalkahbi pura wal & & Alomorfos \\
\hline 9 & Matlalkahbi pura yumhpa & & Alomorfos \\
\hline 10 & $\begin{array}{l}\text { Matawalsip } \\
\text { Matawalsut }\end{array}$ & & Morfema \\
\hline 20 & $\begin{array}{l}\text { Yawanaiska } \\
\text { Yawan aiska kumi }\end{array}$ & $\begin{array}{l}\text { Yawan aiska } \\
\text { Yawanaiska: }\end{array}$ & \\
\hline 100 & $\begin{array}{l}\text { Dusa kum } \\
\text { Lal kum }\end{array}$ & Lexema & \\
\hline
\end{tabular}

\section{Base del sistema de numeración miskita, desde el punto de vista etimológico}

Producto del procesamiento de los instrumentos aplicados, se obtuvo los resultados que se presentan en las figuras del 1 al 3, referidos a la base del sistema de numeración mikitu, desde el punto de vista etimológico.

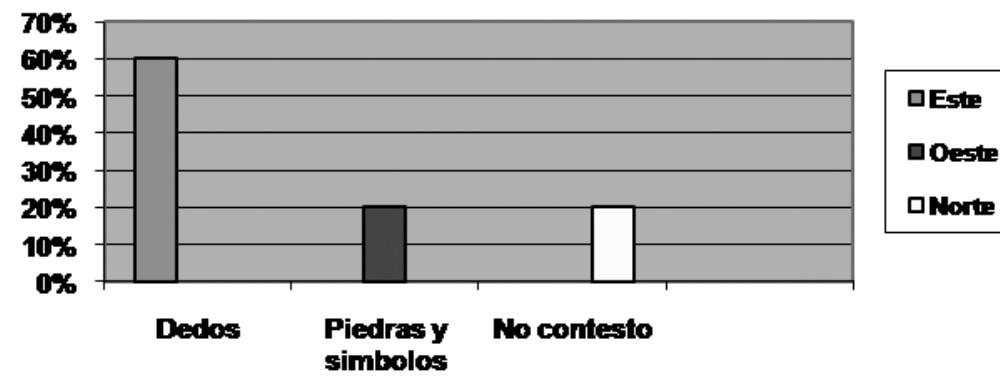

Figura No. 1. Consideración de los docentes sobre la base etimológica del que surge el sistema numérico miskitu. 


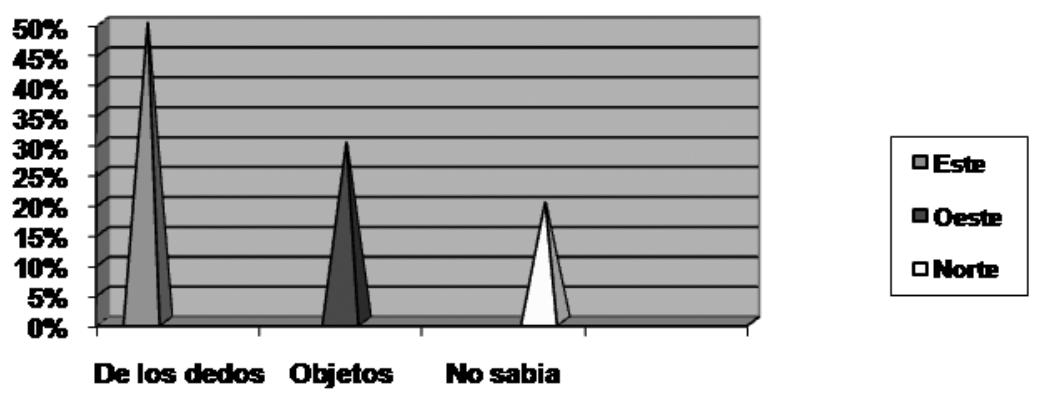

Figura No. 2. Consideración de los padres y madres de familias sobre el surgimiento del sistema numérico miskitu.

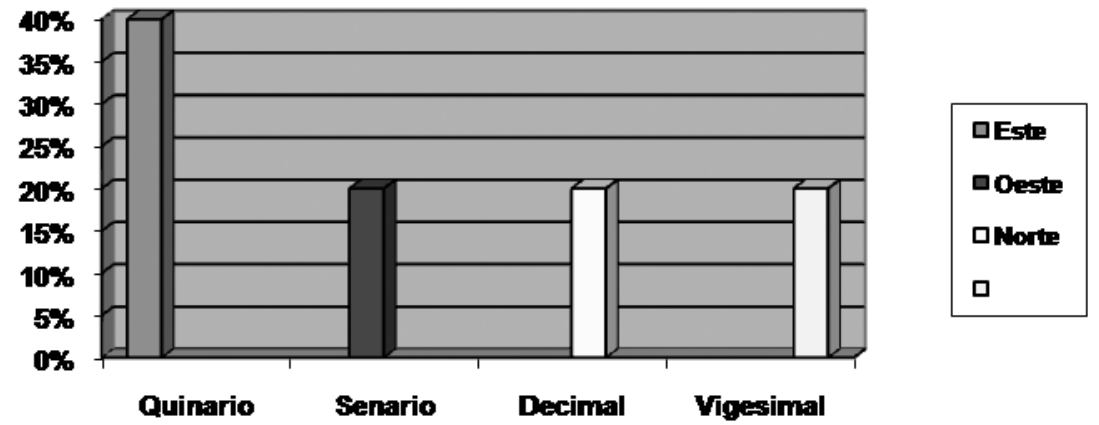

Figura No. 3. Consideración etimológica de los docentes sobre la base del sistema de numeración miskitu.

Referente a las sugerencias de mejoras en los nombres de los números naturales del miskitu, en el caso de los estudiantes, el 90\% seleccionó la propuesta de los nombres neológicos y el 10\% la propuesta de los nombres actuales. Por su parte, el 6o\% de los docentes considera mas fácil los nombres neológicos.

\section{Conclusiones}

\section{Los cambios en los nombres de los números naturales de miskitu}

- La comparación de los diferentes escritos, muestra cambios en los nombres de los números naturales de miskitu, a través de los tiempos. 


\section{Las dificultades lingüísticas en los nombres de los números naturales de miskitu}

- La dificultad en el nombre del número o, 3, 4 es ortográfico, porque la h no deben utilizarse en esos números.

- La dificultad en el nombre del número 5,10 es de morfema: -sip, chip que son de significado dudoso etimológicamente.

- La dificultad en el nombre del número 6 es etimológico, porque al aplicar matlalkahbi como el nombre del número 6 opaca su origen.

- La dificultad en el nombre del número 7, 8, 9 es de alomorfos en donde dos términos de igual significado esta junto: kahbi y pura.

- La dificultad en el nombre del número 20 es de sintaxis, ya que algunos escriben juntos y otros separados el lexema con sus morfemas.

- La dificultad en el nombre del número 100 es de lexema, porque dudan de sus raíces lal o dusa.

\section{Base de sistema de numeración miskitu}

- Los nombres de los números naturales del sistema numeración miskitu en uso actual, tiene indicio de subbase quinaria y base vigesimal.

\section{Propuesta de mejoras en los nombres de los números naturales de mîskitu}

En base la selección de las propuestas y las opiniones, sugerimos la siguiente; propuesta de mejoras de lexemas, morfemas, alomorfos, ortográficos, sintaxis y de fonemas en los nombres de los números naturles de miskitu o a 100, desde el punto de vista etimológico combinado con neologismo. Y según la subbase quinaria y base vigesimal. Esto fortalecerá el proceso de enseñanza de los nombres de los números naturales de miskitu, científicamente ajustados a la evolución de nuestra lengua indígena, es tambien un aporte que contribuirá en los cambios curriculares de la Educación Intercultural Bilingüe.

Cuadro No. 3. Propuesta de mejoras en los nombres de los números naturles de miskitu del 0 a 100, desde el punto de vista etimológico combinado con neologismo.

\begin{tabular}{cll}
\hline No & Nombre actual & Nombre etimo-neológico \\
\hline 0 & Apu & Apu \\
\hline 1 & Kumi & Kum \\
\hline
\end{tabular}




\begin{tabular}{|c|c|c|}
\hline No & Nombre actual & Nombre etimo-neológico \\
\hline 2 & Wal & Wal \\
\hline 3 & Yumhpa & Yumpa \\
\hline 4 & Walhwal & Walwal \\
\hline 5 & Matsip & Mat \\
\hline 6 & Matlalkahbi & Mat kahbi kum \\
\hline 7 & Matlalkahbi pura kumi & Mat kahbi wal \\
\hline 8 & Matlalkahbi pura wal & Mat kahbi yumpa \\
\hline 9 & Matlalkahbi pura yumhpa & Mat kahbi walwal \\
\hline 10 & Matawalsip & Matwal \\
\hline 11 & Matawalsip pura kumi & Mat kahbi kum \\
\hline 12 & Matawalsip pura wal & Mat kahbi wal \\
\hline 13 & Matawalsip pura yumhpa & Mat kahbi yumpa \\
\hline 14 & Matawalsip pura walhwal & Mat kahbi walwal \\
\hline 15 & Matawalsip pura matsip & Matyumpa \\
\hline 16 & Matawalsip pura matlalkahbi & Matyumpa kahbi kum \\
\hline 17 & Matawalsip pura matlalkahbi pura kumi & Matyumpa kahbi wal \\
\hline 18 & Matawalsip pura matlalkahbi pura wal & Matyumpa kahbi yumpa \\
\hline 19 & Matawalsip pura matlalkahbi pura yumhpa & Matyumpa kahbi walwal \\
\hline 20 & Yawan aiska kumi & Ya \\
\hline 21 & Yawan aiska kumi pura kumi & Ya kahbi kum \\
\hline 22 & Yawan aiska kumi pura wal & Ya kahbi wal \\
\hline 23 & Yawan aiska kumi pura yumhpa & Ya kahbi yumpa \\
\hline 24 & Yawan aiska kumi pura walhwal & Ya kahbi walwal \\
\hline 25 & Yawan aiska kumi pura matsip & Ya kahbi mat \\
\hline 26 & Yawan aiska kumi pura matlalkahbi & Ya kahbi mat kahbi kum \\
\hline 27 & $\begin{array}{l}\text { Yawan aiska kumi pura matlalkahbi pura } \\
\text { kumi }\end{array}$ & Ya kahbi mat kahbi wal \\
\hline 28 & Yawan aiska kumi pura matlalkahbi pura wal & Ya kahbi mat kahbi yumpa \\
\hline 29 & $\begin{array}{l}\text { Yawan aiska kumi pura matlalkahbi pura } \\
\text { yumhpa }\end{array}$ & Ya kahbi mat kahbi walwal \\
\hline 30 & Yawan aiska kumi pura matawalsip & Ya kahbi matwal \\
\hline 31 & $\begin{array}{l}\text { Yawan aiska kumi pura matawalsip pura } \\
\text { kumi }\end{array}$ & Ya kahbi matwal kahbi kum \\
\hline
\end{tabular}




\section{REVITALIZACIÓN LINGÜÍSTICA Y CULTURAL}

\begin{tabular}{|c|c|c|}
\hline No & Nombre actual & Nombre etimo-neológico \\
\hline 32 & Yawan aiska lumi pura matawalsip pura wal & Ya kahbi matwal kahbi wal \\
\hline 33 & $\begin{array}{l}\text { Yawan aiska kumi pura matawalsip pura } \\
\text { yumhpa }\end{array}$ & Ya kahbi matwal kahbi yumpa \\
\hline 34 & $\begin{array}{l}\text { Yawan aiska kumi pura matawalsip pura } \\
\text { walhwal }\end{array}$ & Ya kahbi matwal kahbi walwal \\
\hline 35 & $\begin{array}{l}\text { Yawan aiska kumi pura matawalsip pura } \\
\text { matsip }\end{array}$ & Ya kahbi matyumpa \\
\hline 36 & $\begin{array}{l}\text { Yawan aiska kumi pura matawalsip pura } \\
\text { matlalkahbi }\end{array}$ & Ya kahbi matyumpa kahbi kum \\
\hline 37 & $\begin{array}{l}\text { Yawan aiska kumi pura matawalsip pura } \\
\text { matlalkahbi pura kumi }\end{array}$ & Ya kahbi matyumpa kahbi wal \\
\hline 38 & $\begin{array}{l}\text { Yawan aiska kumi pura matawalsip pura } \\
\text { matlalkahbi pura wal }\end{array}$ & Ya kahbi matyumpa kahbi yumpa \\
\hline 39 & $\begin{array}{l}\text { Yawan aiska kumi pura matawalsip pura } \\
\text { matlalkahbi pura yumhpa }\end{array}$ & Ya kahbi matyumpa kahbi walwal \\
\hline 40 & Yawan aiska wal & Yawal \\
\hline 41 & Yawan aiska wal pura kumi & Yawal kahbi kum \\
\hline 42 & Yawan aiska wal pura wal & Yawal kahbi wal \\
\hline 43 & Yawan aiska wal pura yumhpa & Yawal kahbi yumpa \\
\hline 44 & Yawan aiska wal pura walhwal & Yawal kahbi walwal \\
\hline 45 & Yawan aiska wal pura matsip & Yawal kahbi mat \\
\hline 46 & Yawan aiska wal pura matlalkahbi & Yawal kahbi mat kahbi kum \\
\hline 47 & Yawan aiska wal pura matlalkahbi pura kumi & Yawal kahbi mat kahbi wal \\
\hline 48 & Yawan aiska wal pura matlalkahbi pura wal & Yawal kahbi mat kahbi yumpa \\
\hline 49 & $\begin{array}{l}\text { Yawan aiska wal pura matlalkahbi pura } \\
\text { yumhpa }\end{array}$ & Yawal kahbi mat kahbi walwal \\
\hline 50 & Yawan aiska wal pura matawalsip & Yawal kahbi matwal \\
\hline 51 & Yawan aiska wal pura matawalsip pura kumi & Yawal kahbi matwal kahbi kum \\
\hline 52 & Yawan aiska wal pura matawalsip pura wal & Yawal kahbi matwal kahbi wal \\
\hline 53 & $\begin{array}{l}\text { Yawan aiska wal pura matawalsip pura } \\
\text { yumhpa }\end{array}$ & Yawal kahbi matwal kahbi yumpa \\
\hline 54 & $\begin{array}{l}\text { Yawan aiska wal pura matawalsip pura wal- } \\
\text { hwal }\end{array}$ & Yawal kahbi matwal kahbi walwal \\
\hline 55 & $\begin{array}{l}\text { Yawan aiska wal pura matawalsip pura mat- } \\
\text { sip }\end{array}$ & Yawal kahbi matyumpa \\
\hline
\end{tabular}




\begin{tabular}{|c|c|c|}
\hline No & Nombre actual & Nombre etimo-neológico \\
\hline 56 & $\begin{array}{l}\text { Yawan aiska wal pura matawalsip pura mat- } \\
\text { lalkahbi }\end{array}$ & Yawal kahbi matyumpa kahbi kum \\
\hline 57 & $\begin{array}{l}\text { Yawan aiska wal pura matawalsip pura mat- } \\
\text { lalkahbi pura kumi }\end{array}$ & Yawal kahbi matyumpa kahbi wal \\
\hline 58 & $\begin{array}{l}\text { Yawan aiska wal pura matawalsip pura mat- } \\
\text { lalkahbi pura wal }\end{array}$ & Yawal kahbi matyumpa kahbi yumpa \\
\hline 59 & $\begin{array}{l}\text { Yawan aiska wal pura matawalsip pura mat- } \\
\text { lalkahbi pura yumhpa }\end{array}$ & Yawal kahbi matyumpa kahbi walwal \\
\hline 60 & Yawan aiska yumhpa & Yayumpa \\
\hline 61 & Yawan aiska yumhpa pura kumi & Yayumpa kahbi kum \\
\hline 62 & Yawan aiska yumhpa pura wal & Yayumpa kahbi wal \\
\hline 63 & Yawan aiska yumhpa pura yumhpa & Yayumpa kahbi yumpa \\
\hline 64 & Yawan aiska yumhpa pura walhwal & Yayumpa kahbi walwal \\
\hline 65 & Yawan aiska yumhpa pura matsip & Yayumpa kahbi mat \\
\hline 66 & Yawan aiska yumhpa pura matlalkahbi & Yayumpa kahbi mat kahbi kum \\
\hline 67 & $\begin{array}{l}\text { Yawan aiska yumhpa pura matlalkahbi pura } \\
\text { kumi }\end{array}$ & Yayumpa kahbi mat kahbi wal \\
\hline 68 & $\begin{array}{l}\text { Yawan aiska yumhpa pura matlalkahbi pura } \\
\text { wal }\end{array}$ & Yayumpa kahbi mat kahbi yumpa \\
\hline 69 & $\begin{array}{l}\text { Yawan aiska yumhpa pura matlalkahbi pura } \\
\text { yumhpa }\end{array}$ & Yayumpa kahbi mat kahbi walwal \\
\hline 70 & Yawan aiska yumhpa pura matawalsip & Yayumpa kahbi matwal \\
\hline 71 & $\begin{array}{l}\text { Yawan aiska yumhpa pura matawalsip pura } \\
\text { kumi }\end{array}$ & Yayumpa kahbi matwal kahbi kum \\
\hline 72 & $\begin{array}{l}\text { Yawan aiska yumhpa pura matawalsip pura } \\
\text { wal }\end{array}$ & Yayumpa kahbi matwal kahbi wal \\
\hline 73 & $\begin{array}{l}\text { Yawan aiska yumhpa pura matawalsip pura } \\
\text { yumhpa }\end{array}$ & Yayumpa kahbi matwal kahbi yumpa \\
\hline 74 & $\begin{array}{l}\text { Yawan aiska yumhpa pura matawalsip pura } \\
\text { walhwal }\end{array}$ & Yayumpa kahbi matwal kahbi walwal \\
\hline 75 & $\begin{array}{l}\text { Yawan aiska yumhpa pura matawalsip pura } \\
\text { matsip }\end{array}$ & Yayumpa kahbi matyumpa \\
\hline 76 & $\begin{array}{l}\text { Yawan aiska yumhpa pura matawalsip pura } \\
\text { matlalkahbi }\end{array}$ & $\begin{array}{l}\text { Yayumpa kahbi matyumpa kahbi } \\
\text { kum }\end{array}$ \\
\hline 77 & $\begin{array}{l}\text { Yawan aiska yumhpa pura matawalsip pura } \\
\text { matlalkahbi pura kumi }\end{array}$ & Yayumpa kahbi matyumpa kahbi wal \\
\hline
\end{tabular}




\section{REVITALIZACIÓN LINGÜÍSTICA Y CULTURAL}

\begin{tabular}{|c|c|c|}
\hline No & Nombre actual & Nombre etimo-neológico \\
\hline 78 & $\begin{array}{l}\text { Yawan aiska yumhpa pura matawalsip pura } \\
\text { matlalkahbi pura wal }\end{array}$ & $\begin{array}{l}\text { Yayumpa kahbi matyumpa kahbi } \\
\text { yumpa }\end{array}$ \\
\hline 79 & $\begin{array}{l}\text { Yawan aiska yumhpa pura matawalsip pura } \\
\text { matlalkahbi pura yumhpa }\end{array}$ & $\begin{array}{l}\text { Yayumpa kahbi matyumpa kahbi } \\
\text { walwal }\end{array}$ \\
\hline 80 & Yawan aiska walhwal & Yawalwal \\
\hline 81 & Yawan aiska walhwal pura kumi & Yawalwal kahbi kum \\
\hline 82 & Yawan aiska walhwal pura wal & Yawalwal kahbi wal \\
\hline 83 & Yawan aiska walhwal pura yumhpa & Yawalwal kahbi yumpa \\
\hline 84 & Yawan aiska walhwal pura walhwal & Yawalwal kahbi walwal \\
\hline 85 & Yawan aiska walhwal pura matsip & Yawalwal kahbi mat \\
\hline 86 & Yawan aiska walhwal pura matlalkahbi & Yawalwal kahbi mat kahbi kum \\
\hline 87 & $\begin{array}{l}\text { Yawan aiska walhwal pura matlalkahbi pura } \\
\text { kumi }\end{array}$ & Yawalwal kahbi mat kahbi wal \\
\hline 88 & $\begin{array}{l}\text { Yawan aiska walhwal pura matlalkahbi pura } \\
\text { wal }\end{array}$ & Yawalwal kahbi mat kahbi yumpa \\
\hline 89 & $\begin{array}{l}\text { Yawan aiska walhwal pura matlalkahbi pura } \\
\text { yumhpa }\end{array}$ & Yawalwal kahbi mat kahbi walwal \\
\hline 90 & Yawan aiska walhwal pura matawalsip & Yawalwal kahbi matwal \\
\hline 91 & $\begin{array}{l}\text { Yawan aiska walhwal pura matawalsip pura } \\
\text { kumi }\end{array}$ & Yawalwal kahbi matwal kahbi kum \\
\hline 92 & $\begin{array}{l}\text { Yawan aiska walhwal pura matawalsip pura } \\
\text { wal }\end{array}$ & Yawalwal kahbi matwal kahbi wal \\
\hline 93 & $\begin{array}{l}\text { Yawan aiska walhwal pura matawalsip pura } \\
\text { yumhpa }\end{array}$ & Yawalwal kahbi matwal kahbi yumpa \\
\hline 94 & $\begin{array}{l}\text { Yawan aiska walhwal pura matawalsip pura } \\
\text { walhwal }\end{array}$ & Yawalwal kahbi matwal kahbi walwal \\
\hline 95 & $\begin{array}{l}\text { Yawan aiska walhwal pura matawalsip pura } \\
\text { matsip }\end{array}$ & Yawalwal kahbi matyumpa \\
\hline 96 & $\begin{array}{l}\text { Yawan aiska walhwal pura matawalsip pura } \\
\text { matlalkahbi }\end{array}$ & $\begin{array}{l}\text { Yawalwal kahbi matyumpa kahbi } \\
\text { kum }\end{array}$ \\
\hline 97 & $\begin{array}{l}\text { Yawan aiska walhwal pura matawalsip pura } \\
\text { matlalkahbi pura kumi }\end{array}$ & Yawalwal kahbi matyumpa kahbi wal \\
\hline 98 & $\begin{array}{l}\text { Yawan aiska walhwal pura matawalsip pura } \\
\text { matlalkahbi pura wal }\end{array}$ & $\begin{array}{l}\text { Yawalwal kahbi matyumpa kahbi } \\
\text { yumpa }\end{array}$ \\
\hline 99 & $\begin{array}{l}\text { Yawan aiska walhwal pura matawalsip pura } \\
\text { matlalkahbi pura yumhpa }\end{array}$ & $\begin{array}{l}\text { Yawalwal kahbi matyumpa kahbi } \\
\text { walwal }\end{array}$ \\
\hline 100 & Dusa kumi & Dus \\
\hline
\end{tabular}




\section{Lista de Referencias}

Cárdenas, C. y David, F. (1993). Kulki Sakaia Sinska 1². MECD. Managua, Nicaragua.

Iglesia Morava (2009). Yua Banira Aisi Kaikaia Bila. Puerto Cabezas,RAAN.

OCEANO (2000). Enciclopedia Didáctica de Matemática. Barcelona, España: MMIV Editorial Oceano.

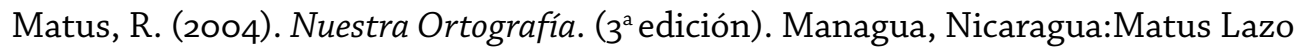
Ediciones..

Matus, Roger (2007). Español 2. (5 ${ }^{\text {ta }}$ edición). Managua, Nicaragua: Matus Lazo Ediciones.

M. Padilla (2001). Dossier de Miskitu I. Puerto cabezas Nicaragua.

MED (1987). Domina la Ortografía. Managua, Nicaragua.

MECD (2004). Texto 5, III Nivel Alternativo. Managua, Nicaragua.

Sociedad Bíblica (1974). Dawan Bila Testament Raya. Managua, Nicaragua.

Sociedad Bíblica (1999). Dawan Bila Aiska. Managua, Nicaragua.

URACCAN (2001). Dossier de Lingüística General Aplicada a la E.I.B.

Bilwi Puerto Cabezas, Nicaragua.

Urbina, Miguel (2004). Gramática Pedagógica Miskitu. Managua, Nicaragua: Multigrafic.

\section{Recursos electrónicos}

http//.www.es.wikipedia.org/sistema-quinario

http//htm-rincón del vago.com/morfologico-1htm

http//htm-rincón del vago.com/neologismo-1htn 